\title{
Avaliação da resistência à corrosão de eletrodepósitos de liga ZnFe sobre aço tratado com filmes contendo sais de terras raras e silanos BTSE, BTESPTS
}

\author{
Evaluation of corrosion resistance of steel \\ coated with zinc-iron alloy electrodeposits \\ containing BTSE, BTESPTS and rare earth \\ salts as conversion films
}

Faculdade de Engenharia Mecânica, UNICAMP, C.P. 6122, 13083-970 Campinas - SP msantos22@zipmail.com.br; célia@ fem.unicamp.br

\begin{abstract}
RESUMO
O presente trabalho tem como objetivo avaliar a resistência a corrosão do aço revestido com eletrodepósito de liga $\mathrm{ZnFe}$ tratado com filmes constituídos de uma pré-camada obtida através de solução de sais de terras raras (Ce ou La) acrescida de filmes de silanos bis 1,2-[trietoxissilil]etano) (BTSE) e/ou bis(3-[trietoxissilil]-propil) - tetrassulfeto (BTESPTS). Esses sistemas foram avaliados através das Curvas de Polarização, Espectroscopia de Impedância Eletroquímica (EIE), Microscopia Eletrônica de Varredura (MEV) e Espectrometria de Energia Dispersiva (EDS). Os resultados de EIE e polarização indicaram que os sistemas estudados foram eficientes contra corrosão e que seu desempenho depende do tipo de cátion do sal. O sistema $\mathrm{ZnFe}+\mathrm{Ce}+\mathrm{BTSE}+\mathrm{BTESPTS}$ mostrou o melhor resultado contra corrosão.
\end{abstract}

Palavras chaves: corrosão, revestimento de liga $\mathrm{ZnFe}$, silano, terras raras

\begin{abstract}
This study aims to evaluate the corrosion resistance of steel coated with $\mathrm{ZnFe}$ alloy electrodeposits treated with films consisted of a pre-layer obtained through immersion in a rare earth salt solution using Ce or La elements plus a silane thin film of BTSE (bis 1, 2 - [triethoxysilyl]) ethane) and / or BTESPTS (bis-(3 - [triethoxysilyl]-propyl) - tetrassulfeto). These systems were evaluated by polarization curves, electrochemical impedance spectroscopy (EIS), scanning electron microscopy (SEM) and energy dispersive spectroscopy (EDS). The polarization and EIS results indicated that the studied systems were effective against corrosion and that their performance depends on the type of cation salt. The $\mathrm{ZnFe}+\mathrm{Ce}++\mathrm{BTSE}$ BTESPTS system proved to be the best result against corrosion.
\end{abstract}

Keywords: corrosion, zin-iron alloy coating, silane, rare earth.

\section{INTRODUÇÃO}

A utilização de ligas de zinco como forma de proteção catódica em aços ocorre porque o zinco é menos nobre que o ferro, dessa forma, quando os dois metais estão em contato no mesmo meio agressivo, o ferro é protegido à custa da dissolução eletrolítica do zinco. Além disso, os aços revestidos com ligas de zinco possuem numerosas e variadas aplicações, pois são utilizados na construção civil, em dutos para distribuição de água, em equipamentos de refrigeração, na indústria automobilística, em estruturas metálicas, equipamentos elétricos e em outras aplicações [1].

Os depósitos comerciais que apresentam teores de ferro de 0,3 a $2 \%$ requerem, no entanto, tratamento posterior de cromatização, que inibe o aparecimento da corrosão branca, normalmente presente em aços revestidos com ligas de $\mathrm{Zn}$. Porém, esse processo de passivação tem como base cromatos que possuem cromo hexavalente $\left(\mathrm{Cr}^{+6}\right)$, que é tóxico; o que contraria as tendências atuais de desenvolvimento ambiental sustentável, que exige o aprimoramento de técnicas e metodologias com a geração cada vez menor de resíduos tóxicos [2]. 
Como exemplo dessa tendência tem-se a diretiva 2000/53/EC do Parlamento Europeu "end-of vehicle" que definiu novos padrões de materiais e processos utilizados na indústria automotiva e, onde partes contendo $\mathrm{Cr}^{6+}$ não são mais aceitas desde 2007 [3].

As atuais alternativas à substituição do $\mathrm{Cr}^{+6}$ em processos de passivação, tais como a deposição de molibdatos, a preparação de materiais híbridos organo-inorgânicos através do processo sol-gel e a deposição de filmes contendo sais de terras raras, apresentam prós e contras, quando propriedades como resistência à corrosão, adesão de revestimentos orgânicos, resistência à fadiga, desempenho e controle de qualidade, são considerados. Entretanto, algumas dessas alternativas apresentam potencial de uso, caso algumas melhorias sejam feitas [1, 4-14].

Os estudos desenvolvidos por Ferreira, et al. [15], PENG e MAN [16], Aramaki K. [17], TRABELSI W. et al. [18] e TRABELSI W. et al. [19], registram estudos sobre o tratamento das superfícies de materiais a base de zinco fazendo uso das terras raras (Ce ou La) com objetivo de formar uma camada de passivação juntamente com o siloxano para melhorar a proteção por barreira contra a corrosão. Esses trabalhos (TR+silano) sobre superfícies de zinco apresentaram melhorias na resistência à corrosão e aderência em sistemas pintados.

Levando em consideração o crescente consumo de eletrodepósitos de ligas de zinco pelas indústrias de construção civil, aeronáutica e automotiva, esse estudo procura fornecer não apenas informações sobre uma mono ou dupla camada de silanos que atuem como barreira em processos corrosivos, como também se existe melhoria das propriedades de resistência a corrosão através da adição de inibidores (terras raras), na interface dos filmes de silano e a liga $\mathrm{ZnFe}$ eletrodepositada. A resistência à corrosão destes filmes foi feita através de ensaios eletroquímicos utilizando as técnicas de espectroscopia de impedância eletroquímica e ensaios de polarização.

\section{MATERIAIS E MÉTODOS}

O procedimento experimental foi desenvolvido em quatro etapas básicas que estão detalhadas a seguir e que podem ser sintetizadas da seguinte forma:

1.Eletrodeposição da camada de liga ZnFe sobre aço.

2. Deposição das camadas de terras raras.

3. Deposição das camadas de silanos sobre as camadas de terras raras.

4. Caracterização eletroquímica dos sistemas em estudo

\subsection{Preparação da superfície metálica}

\subsubsection{Eletrodeposição da camada de liga ZnFe sobre aço}

Amostras de aço carbono 1020 passaram por um processo de eletrodeposição de camada de liga ZnFe utilizando-se banhos comerciais e o processo de eletrodeposição por corrente pulsada [20].

\subsubsection{Preparo das pré-camadas contendo sais de terras raras}

Após a eletrodeposição da camada de liga ZnFe a superfície dos corpo-de-prova foi limpa com solução detergente por 2 minutos, seguida de lavagem em água por 30 segundos e lavagem em água destilada e deionizada por mais 30 segundos. Após a limpeza das amostras as mesmas foram imersas em solução de nitrato de cério ou nitrato de lantânio por um período de 3 minutos. Para deposição das pré-camadas de cério e lantânio foram utilizadas soluções contendo sais de $\mathrm{Ce}\left(\mathrm{NO}_{3}\right)_{3}$ ou $\mathrm{La}\left(\mathrm{NO}_{3}\right)_{3}$ na concentração de $0,01 \mathrm{M}$. Após o procedimento de imersão nas soluções de terras raras as amostras foram colocadas em estufa para secagem.[20]

\subsubsection{Preparo das camadas de silanos:}

Após a etapa anterior, as amostras contendo as camadas de sais de terras raras foram imersas em solução de silano por 3 minutos, seguida de secagem em estufa.

Uma solução 5\% de BTSE foi preparada dissolvendo a alíquota correspondente a este volume de silano em uma mistura de água deionizada e álcool etílico na concentração 50/50 (v/v) com pH resultante final de 4,2. A solução foi feita sob agitação constante à temperatura ambiente e hidrolisada por 1 hora antes do uso [21]. Uma solução 5\% de BTESPTS foi preparada dissolvendo a alíquota correspondente a este volume de silano em uma mistura de água deionizada e álcool etílico na concentração 5/95 (v/v) com pH resultante final de 6,5. A solução foi feita sob agitação constante à temperatura ambiente e após o tempo de 10 minutos Cada solução foi preparada na concentração, volume e pHs ótimos para cada silano, obedecendo 
a compatibilidade de cada silano no meio alcoólico considerando dessa forma, sua máxima eficácia dentro das condições impostas pela cinética das reações.

Os valores dos $\mathrm{pH}$ das soluções foram ajustados com soluções de ácido acético $1 \mathrm{M}$ e/ou hidróxido de amônio $1 \mathrm{M}$.

\subsubsection{Aplicação das soluções de silano sobre as pré-camadas de sais de terras raras}

A tabela 1 apresenta a nomenclatura das amostras estudadas.

Tabela 1: Amostras e respectivos tratamentos sofridos

\begin{tabular}{l|l}
\hline \multicolumn{1}{c|}{ Amostra } & \multicolumn{1}{c}{ Tratamento } \\
\hline $\mathrm{ZnFe}+\mathrm{La}+\mathrm{BTSEPTS}$ & Imersão em solução de sais de terras raras $-\mathrm{La}$, seguida da aplicação do silano BTSEPTS \\
$\mathrm{ZnFe}+\mathrm{Ce}+\mathrm{BTSEPTS}$ & Imersão em solução de sais de terras raras $-\mathrm{Ce}$, seguida da aplicação do silano BTSEPTS \\
$\mathrm{ZnFe}+\mathrm{La}+\mathrm{BTSE}+$ & $\begin{array}{l}\text { Imersão em solução de sais de terras raras }-\mathrm{La} \text {, seguida da aplicação dos silanos BTSE + } \\
\text { BTSEPTS } \\
\mathrm{BnFe}+\mathrm{Ce}+\mathrm{BTSE}+ \\
\text { BTSEPTS }\end{array}$ \\
\hline
\end{tabular}

A imersão na solução de sais de terras raras foi feita por um período de 4,5 minutos com posterior secagem em estufa a $100^{\circ} \mathrm{C}$ por um período de 40 minutos seguida de secagem à temperatura ambiente por 24 horas. Para as amostras com deposição do silano BTSEPTS, adotou-se a seguinte metodologia: após a deposição da pré-camadas de sais de terras raras foi feita a imersão do corpo-de-prova na solução do silano durante 3 minutos, seguida de secagem em estufa a $100^{\circ} \mathrm{C}$ durante 40 minutos; após a secagem em estufa a amostra foi mantida à temperatura ambiente por 24 horas.

Para as amostras contendo ambos os silanos a metodologia empregada seguiu as etapas: deposição da précamada de sais de terras raras, seguida da deposição do silano BTSE através da imersão da amostra na solução do silano por 10 minutos, seguida de secagem em estufa a $80^{\circ} \mathrm{C}$ por 10 minutos e posterior acondicionamento em dessecador. Na sequência a amostra contendo as camadas anteriores, foi imersa na solução do silano BTSEPTS por 10 minutos, seguida de cura em estufa a $100^{\circ} \mathrm{C}$ por 40 minutos. Após a retirada da estufa a amostra foi mantida à temperatura ambiente por 24 horas.

Vários autores na literatura como FERREIRA M.G.S. et al. [22], CABRAL A. et al. [23], CABRAL A.M. et al. [24], TRABELSI W. et al. [25], relatam estudos com diferentes tempos de imersão para o silano BTSPTS sobre o substrato de zinco, diferentes tempos de secagem em estufa e de temperaturas que podem variar de $100^{\circ} \mathrm{C}, 120^{\circ} \mathrm{C}$ a temperaturas superiores para as amostras a base de zinco,mostrando bons resultados.

\subsection{Caracterização eletroquímica: espectroscopia de impedância eletroquímica (EIE) e curvas de polarização}

As características eletroquímicas dos sistemas silano/substratos foram estudadas por EIE sobre o potencial de circuito aberto na faixa de frequência de $10^{-2}$ a $10^{5} \mathrm{~Hz}$ e 5 pontos por década, utilizando-se um potenciostato modelo PAR 273A da EG\&G em conjunto com um lock-in modelo 5210. Para o gerenciamento dos ensaios e aquisição dos dados foi utilizado o software PowerSine (PAR) . A amplitude da função senoidal de tensão usada foi de $10 \mathrm{mV}$. A célula de três eletrodos para amostras planas, continha um eletrodo de referência de calomelano saturado (SCE), uma grande grade de platina como contra-eletrodo e eletrodo de trabalho formado pelo substrato de aço revestido da liga $\mathrm{ZnFe}$ com área exposta de $19,6 \mathrm{~cm}^{2}$ revestida pelos filmes de silanos. Uma solução $0,05 \mathrm{M}$ de $\mathrm{NaCl}$ foi usada como eletrólito para caracterizar as camadas.

As curvas de polarização das amostras e da liga $\mathrm{ZnFe}$ foram obtidas em solução de $\mathrm{NaCl}$ 0,05M usando o eletrodo de calomelano saturado como referência e platina como contra eletrodo. As curvas de polarização foram obtidas após 20 minutos de imersão da amostra no eletrólito, para estabilização do potencial de circuito aberto. Após esse período a varredura foi feita inicialmente num intervalo de $-250 \mathrm{mV}$ a $250 \mathrm{mV}$ em relação ao potencial de circuito aberto numa velocidade de $0,166 \mathrm{mV} / \mathrm{s}$. Para separar as curvas de polarização potenciodinâmica anódica e catódica novas curvas, nas mesmas condições, foram levantadas num intervalo de $-1,0 \mathrm{~V}$ a $0,0 \mathrm{~V}$ e $-1,0 \mathrm{~V}$ a $-2,0 \mathrm{~V}$ em relação ao ESCE na velocidade $0,166 \mathrm{mV} / \mathrm{s}$, respectivamente. Os ensaios foram realizados à temperatura ambiente e meio naturalmente aerado. Foram feitos 2 ensaios para cada amostra.

\subsection{Caracterização por Microscopia Eletrônica de Varredura - EDS}


A superfície das amostras foi analisada por EDS utilizando-se um microscópio eletrônico de varredura JEOL JXA-804A, com o intuito de se identificar a presença do depósito sobre a camada de liga $\mathrm{ZnFe}$. Para tanto foi feito o mapeamento dos elementos sobre a superfície das amostras contendo as camadas de depósito $\mathrm{ZnFe}+\mathrm{TR}$ + BTSE + BTESPTS. Para análise foi utilizada uma tensão de aceleração de $20 \mathrm{KeV}$.

\section{RESULTADOS}

Nesta etapa são apresentados os resultados relacionados aos ensaios de resistência à corrosão por EIE para cada um dos filmes aplicados sobre a liga $\mathrm{ZnFe}$ em meio de $\mathrm{NaCl} 0,05 \mathrm{M}$ e na sequência os ensaios de polarização relacionado-os com os resultados das análises de EIE.

Os melhores resultados apresentados por EIE e polarização das amostras serão mostrados com as imagens obtidas por MEV e semi-quantificadas por EDS, antes e depois das 72 horas de ensaio de corrosão em $\mathrm{NaCl} 0,05 \mathrm{M}$.

A tabela 2 apresenta as denominações das amostras para os respectivos filmes e a camada de liga $\mathrm{ZnFe}$. Esta denominação será utilizada no decorrer da apresentação de maneira a facilitar a discussão e o entendimento das explicações e considerações. Os resultados de EIE para as amostras serão apresentados no primeiro tempo de imersão (0 minuto) e após 72 horas de imersão e sempre em comparação com o primeiro momento do ensaio de corrosão para a liga $\mathrm{ZnFe}$.

Tabela 2: Denominação das amostras.

\begin{tabular}{|c|c|}
\hline Amostras & Filmes obtidos a partir de: \\
\hline ZnFe+La+BTSEPTS & $\mathrm{La}_{2} \mathrm{O}_{3}+\mathrm{BTSEPTS}$ \\
\hline ZnFe+Ce+BTSEPTS & $\mathrm{Ce}_{2} \mathrm{O}_{3}$ e CeO \\
\hline ZnFe+La+BTSEPTS \\
\hline ZnFe+Ce+BTSE+ BTSEPTS & $\mathrm{La}_{2} \mathrm{O}_{3}+\mathrm{BTSE}+\mathrm{BTSEPTS}$ \\
\hline $\mathrm{Ce}_{2} \mathrm{O}_{3} \mathrm{e} \mathrm{CeO}_{2}+\mathrm{BTSE}+\mathrm{BTSEPTS}$ \\
\hline
\end{tabular}

As amostras foram denominadas, em função das soluções que contém silano, independentes do $\mathrm{pH}$ das soluções como:

BTSE: 5\% em volume do silano bis (1,2 - [trietoxissilil]etano) em solução 50/50 de água/etanol.

BTSEPTS: 5\% em volume do silano viniltrimetoxissilano em solução 5/95 de água/etanol.

As Figuras 1 e 2 apresentam os diagramas de Bode $\mathrm{Z}$ e Bode Fase para a liga $\mathrm{ZnFe}$, a liga $\mathrm{ZnFe}$ revestida somente com o filme de BTSESPT, e a liga de ZnFe revestida pela soma de BTSE com BTSESPT. Nota-se também, que os revestimentos mencionados estão sobre dois diferentes filmes intermediários, contendo na Figura 1 óxidos de cério e na Figura 2 óxido de lantânio. O comportamento das amostras contendo apenas a pré-camada de sais de terras raras foi colocado como referência [20] com o objetivo de comparar o efeito da presença da camada de silano.

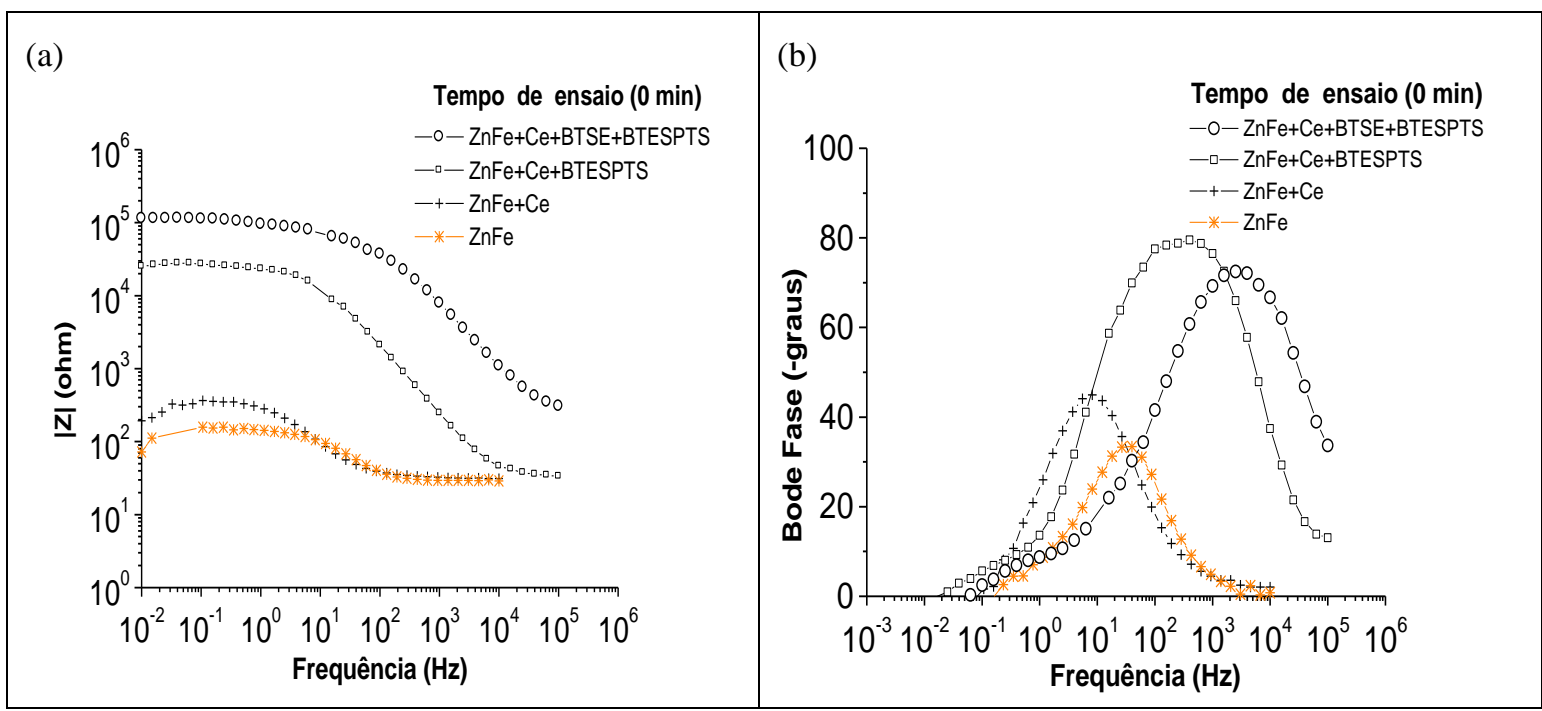

Figura 1: Diagramas de Bode para os sistemas com camada intermediária contendo Ce e para a liga ZnFe no primeiro tempo de ensaio ( $\mathrm{t}=0 \mathrm{~min}$.). 
Para as amostras analisadas, isto é, as amostras contendo os filmes intermediários de óxidos de terras raras revestidos por BTESPT somente e por BTESPT+BTSE, apresentam para o tempo zero minuto de imersão em solução salina $0,05 \mathrm{M}$, valores de $|\mathrm{Z}|$ de $10^{4}$ e $10^{5}$ ohms, respectivamente. Nas Figuras $1 \mathrm{~b}$ e $2 \mathrm{~b}$ observa-se uma constante de tempo entre $10^{1} \mathrm{e} 10^{4} \mathrm{~Hz}$ para a amostra contendo BTESPTS somente a outra entre $10^{3} \mathrm{e}$ $10^{5} \mathrm{~Hz}$ para a amostra de BTESPT+BTSE.

Em um primeiro momento, ou seja, no tempo zero minuto de imersão, os resultados de EIE indicam os sistemas representados pelas amostras $\mathrm{ZnFe}+\mathrm{Ce}+\mathrm{BTSE}+\mathrm{BTESPTS}$ e $\mathrm{ZnFe}+\mathrm{La}+\mathrm{BTSE}+\mathrm{BTESPTS}$, como as melhores em termos de proteção por barreira contra corrosão.

Para confirmação, no entanto, desse comportamento eletroquímico todas as amostras foram submetidas a analises temporal de imersão em $\mathrm{NaCl}$ 0,05M.
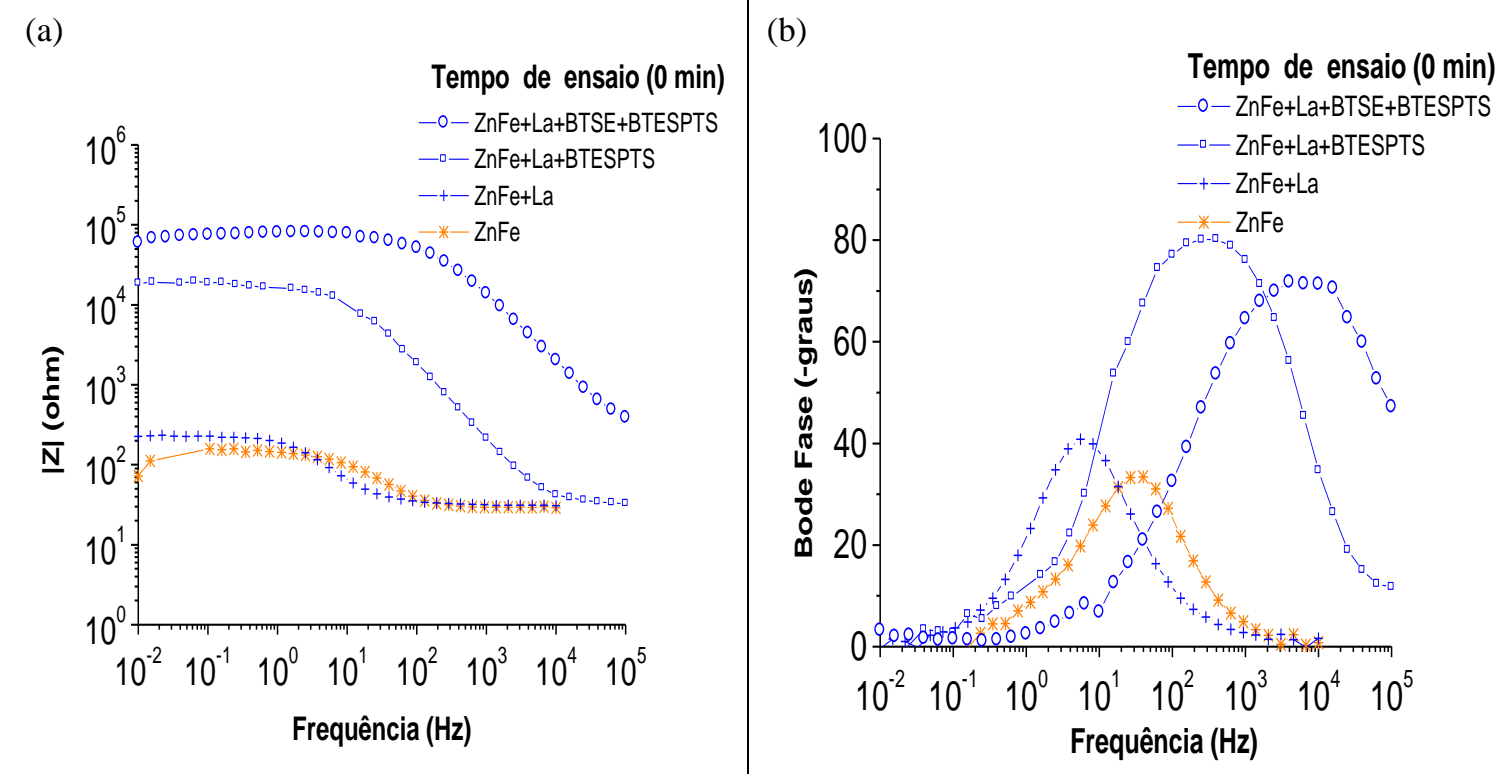

Figura 2: Diagramas de Bode para os sistemas com camada intermediária contendo La e para a liga ZnFe no primeiro tempo de ensaio ( $\mathrm{t}=0$ min.).

As Figuras 3 e 4 mostram os resultados da EIE para todas as amostras apresentadas na Tabela 2 no tempo de 72 horas de imersão em solução salina.

Após 72 horas de imersão em solução de $\mathrm{NaCl}$ é possível observar o efeito protetor contra a corrosão dos sistemas representados pelas amostras $\mathrm{ZnFe}+\mathrm{Ce}+\mathrm{BTESPTS}$ e $\mathrm{ZnFe}+\mathrm{Ce}+\mathrm{BTSE}+\mathrm{BTESPTS}$, que apresentam $|Z|$ em torno de $2.0 \times 10^{4}$ e $7.0 \times 10^{5}$ ohms respectivamente, ou seja, não se observa alterações significativas nas características protetoras dos filmes.

A Figura 4 apresenta para as amostras $\mathrm{ZnFe}+\mathrm{La}+\mathrm{BTESPT}$ e $\mathrm{ZnFe}+\mathrm{La}+\mathrm{BTSE}+\mathrm{BTESPT}$ valores para $|\mathrm{Z}|$ de $4.0 \times 10^{3}$ e $5.0 \times 10^{4} \mathrm{ohms}$, respectivamente, indicando o segundo sistema como o mais protetor após 72 horas de imersão em solução de $\mathrm{NaCl}$ 0,05M. Na Figura 4b, observa-se na amostra ZnFe+La+BTSE+BTESPT o surgimento de três constantes de tempo em baixas, médias e altas freqüências, o que indica a presença do primeiro silano depositado, ou seja, o BTSE. Pode ser observado, comparativamente, na Figura 3b, que após o mesmo tempo de imersão em solução salina, ocorre a presença de duas constantes de tempo para o sistema contendo os dois silanos sendo uma corcova em média e a outra em baixa frequência.

O comportamento eletroquímico da liga ZnFe e das amostras analisadas anteriormente por EIE, foram avaliados através da técnica de polarização potenciodinâmica. FERREIRA M.G.S ET al. [22] também realizou ensaios de EIE e ensaios de polarização potenciodinâmica para silanos mais terras raras sobre o aço galvanizado e verificou uma diminuição na corrente de corrosão, confirmando assim o efeito barreira. Novamente as amostras contendo apenas a camada de sais de terras raras são inseridas no gráfico a título de comparação [20] 


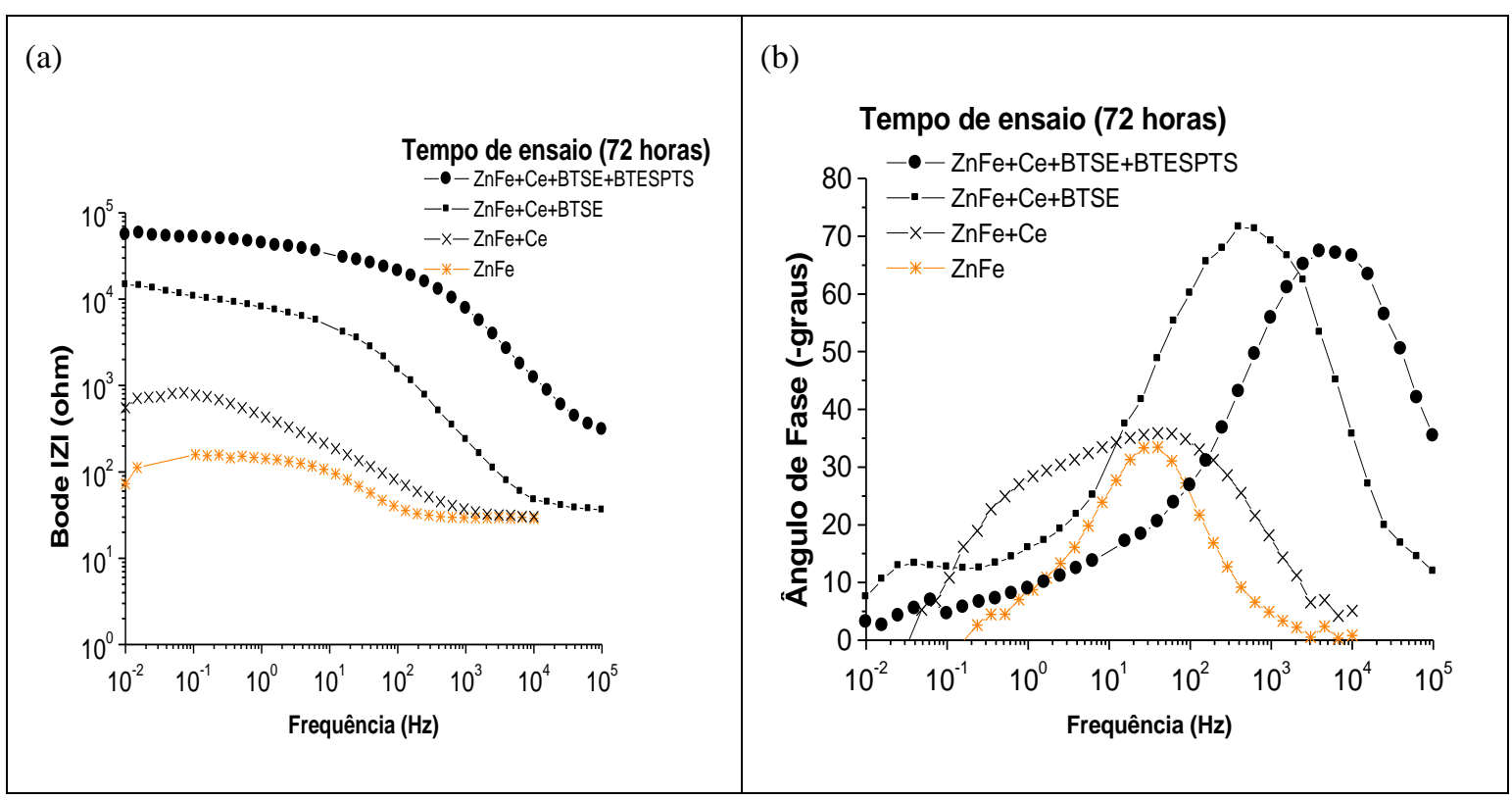

Figura 3: Diagramas de Bode para os sistemas com camada intermediária contendo Ce e para a liga ZnFe em 72 horas de ensaio ( $\mathrm{t}=72$ horas).

(a)

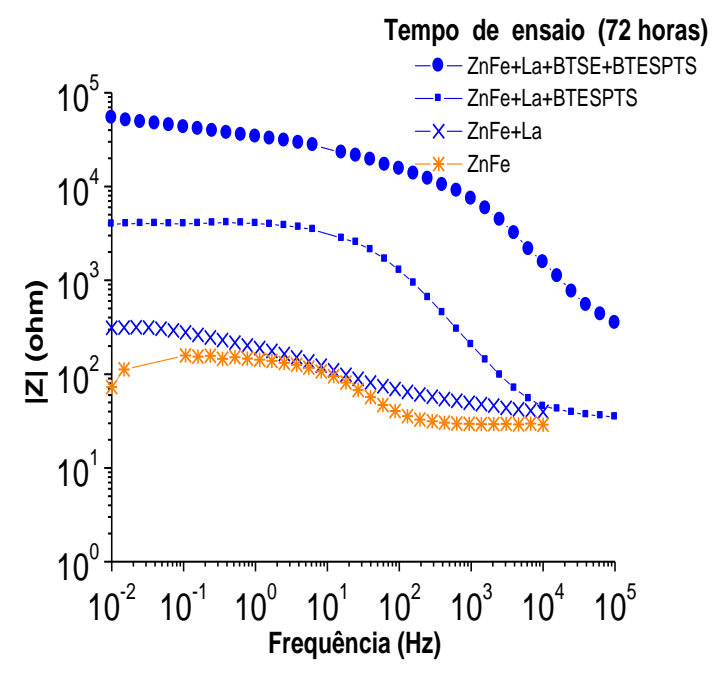

(b)

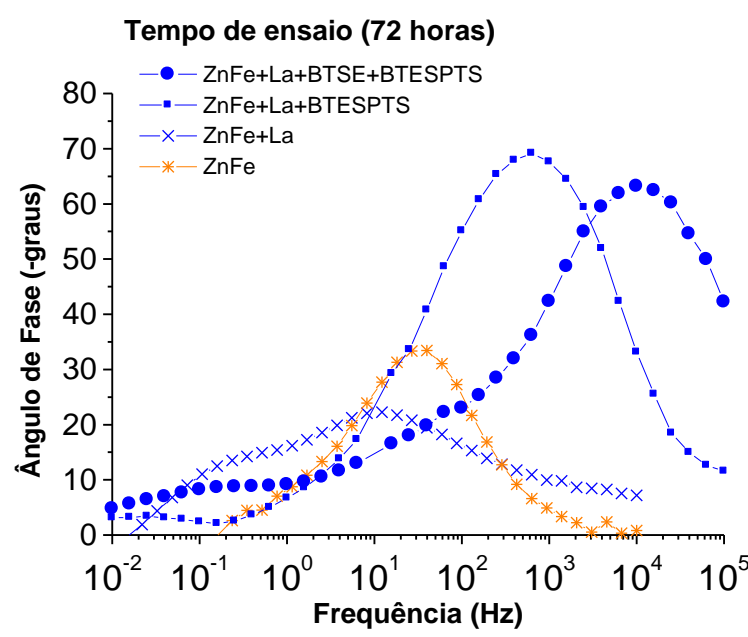

Figura 4: Diagramas de Bode para os sistemas com camada intermediária contendo La e para a liga ZnFe em 72 horas de ensaio ( $\mathrm{t}=72$ horas).

A Figura 5 apresenta as curvas de polarização anódicas e catódicas para a liga ZnFe e para as amostras, $\mathrm{ZnFe}+\mathrm{Ce}+\mathrm{BTSE}, \quad \mathrm{ZnFe}+\mathrm{La}+\mathrm{BTSE}, \quad \mathrm{ZnFe}+\mathrm{Ce}+\mathrm{BTESPTS}, \quad$ ZnFe+La+BTESPTS, $\mathrm{ZnFe}+\mathrm{Ce}+\mathrm{BTSE}+\mathrm{BTESPTS}$ e $\mathrm{ZnFe}+\mathrm{La}+\mathrm{BTSE}+\mathrm{BTESPTS}$.

Os resultados mostram que os tratamentos realizados provocaram o deslocamento do ramo anódico das curvas de polarização em torno de 3 vezes na corrente de resposta; entretanto, a diminuição da corrente na região catódica foi mais marcante, havendo a diminuição, em termos de corrente, na ordem de 1.000 vezes para os sistemas contendo as camadas terras raras + silanos.

A fim de conhecer melhor o comportamento das amostras que apresentaram redução significativa de corrente resultante na região catódica das curvas de polarização, os ensaios de polarização anódica e catódica foram realizados separadamente para as melhores amostras, ou seja, ZnFe+La+BTSE+BTESPTS , $\mathrm{ZnFe}+\mathrm{Ce}+\mathrm{BTSE}+\mathrm{BTESPTS}$ e para a liga $\mathrm{ZnFe}$ mostrados nas Figuras 6 e 7 respectivamente. 


\section{Módulo de Tafel}

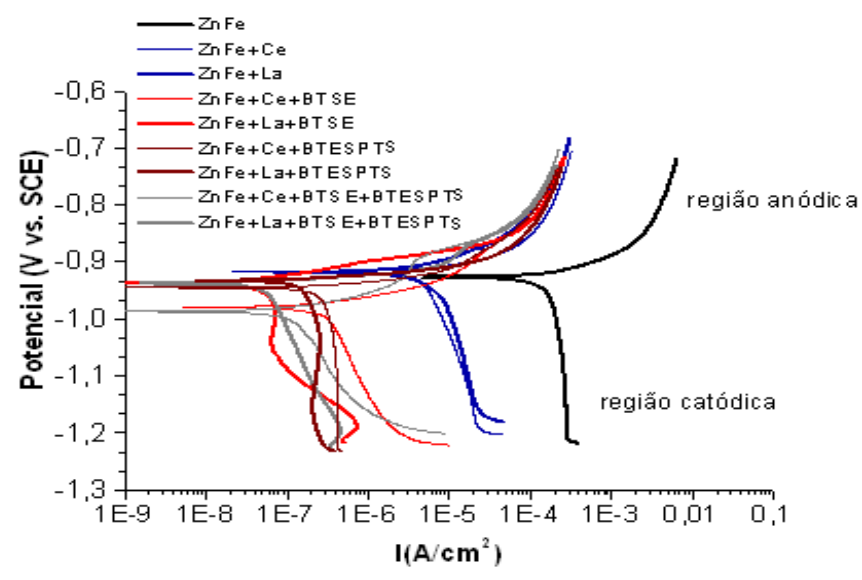

Figura 5: Curvas de polarização da liga $\mathrm{ZnFe}$ e de diferentes amostras ensaiadas em solução de $\mathrm{NaCl}$ 0,05M. (tempo de ensaio $=20$ minutos).

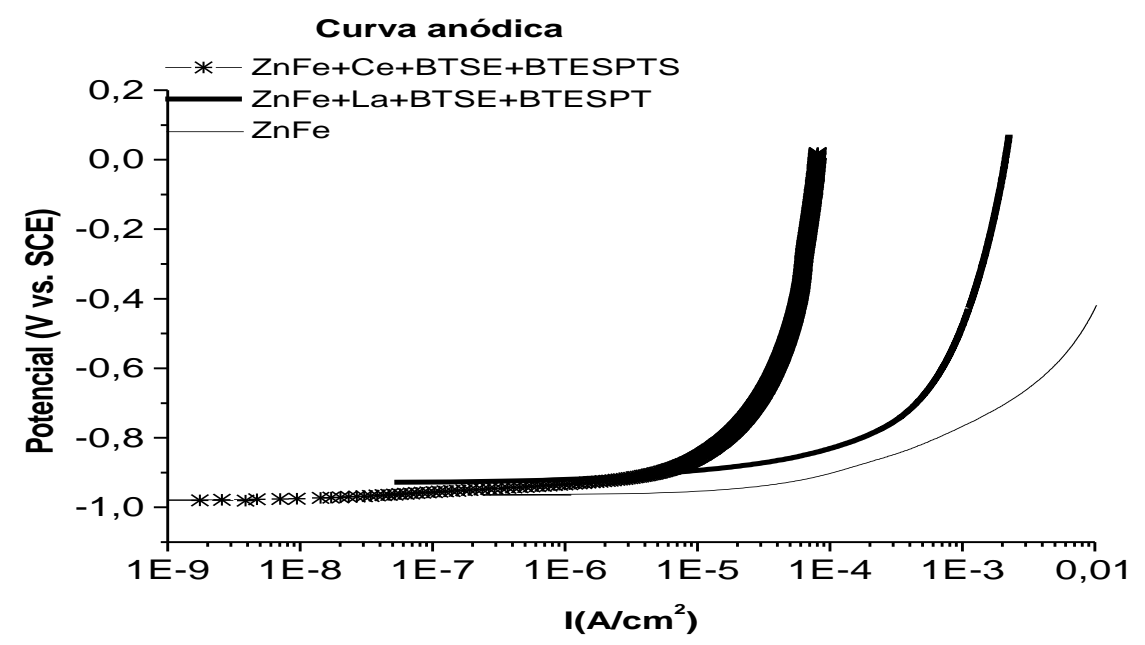

Figura 6: Curva anódica para a a liga ZnFe e para as amostras ZnFe+Ce+BTSE+BTSPTS e ZnFe+La+BTSE+BTSPTS ensaiadas em solução de $\mathrm{NaCl}$ 0,05M (tempo de ensaio=20 minutos).

A Figura 6 apresenta a curva anódica para a liga ZnFe e para as amostras ZnFe+Ce+BTSE+BTESPTS e $\mathrm{ZnFe}+\mathrm{La}+\mathrm{BTSE}+\mathrm{BTESPTS}$.

As curvas mostram redução de corrente de resposta na seguinte ordem: $\mathrm{ZnFe}>\mathrm{ZnFe}+\mathrm{La}+\mathrm{BTSE}+$ BTESPTS > ZnFe + Ce+ BTSE + BTESPTS. Nota-se que ao se elevar a faixa de potencial anódico o comportamento da corrente se modifica, com deslocamento no eixo de corrente em até três décadas se compararmos a amostra $\mathrm{ZnFe}+\mathrm{Ce}+\mathrm{BTSE}+\mathrm{BTESPTS}$ em relação a liga ZnFe, o que sugere a inibição do processo de dissolução anódica da liga $\mathrm{ZnFe}$.

A Figura 7 apresenta as reações catódicas ocorridas na superfície da liga ZnFe e das amostras tratadas com terras raras mais os silanos BTSE e BTESPTS. 


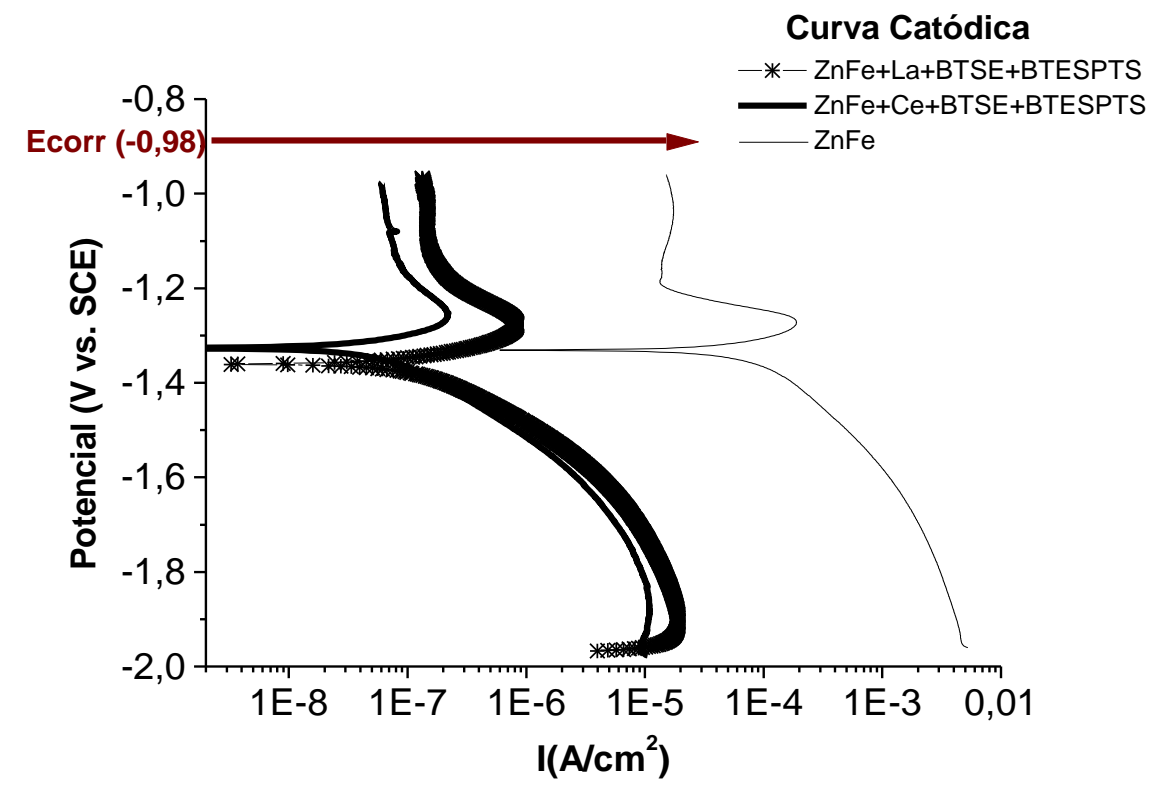

Figure 7: Curva catódica para o substrato de ZnFe e amostras ZnFe+La+BTSE+BTESPTS e $\mathrm{ZnFe}+\mathrm{La}+\mathrm{BTSE}+\mathrm{BTESPTS}$ ensaiadas em solução de $\mathrm{NaCl}$ 0,05M. (tempo de ensaio=20 minutos).

As curvas mostram deslocamento do ramo catódico no eixo da corrente resultante na seguinte ordem: $\mathrm{ZnFe} \mathrm{>}$ $\mathrm{ZnFe}+\mathrm{La}+\mathrm{BTSE}+\mathrm{BTESPTS}>\mathrm{ZnFe}+\mathrm{Ce}+\mathrm{BTSE}+\mathrm{BTESPTS}$. Levando em consideração as curvas de polarização das Figuras 5 e 7 e suas indicações de domínio catódico para as amostras, pode-se dizer que a inibição catódica ocorre por causa de um sinergismo entre o efeito físico de barreira dos filmes de silano responsáveis por reduzir a área ativa da superfície e os filmes de óxidos de terras raras que atuam sobre os sítios catódicos criando uma barreira ao processo de redução do oxigênio. ARAMAKI, K., [9] TRABELSI W et al. [19], CABRAL A.M, et al. [24] e ZHU and OOIJ W.J. [26], observaram os deslocamentos do ramo catódico no eixo da corrente catódica resultante em seus sistemas estudados, corroborando com os resultados apresentados.

As Figuras 8 e 9 apresentam os resultados de mapeamento dos elementos fornecidos pela análise por EDS para as amostras de ZnFe + Ce + BTSE + BTESPTS e ZnFe + La + BTSE + BTESPTS onde o Si presente é, possivelmente, proveniente das ligações Si-O-Si, Si-O-C, Si-O-Fe e Si-O-Zn, o S da molécula de BTESPTS e o Ce e La das terras raras.

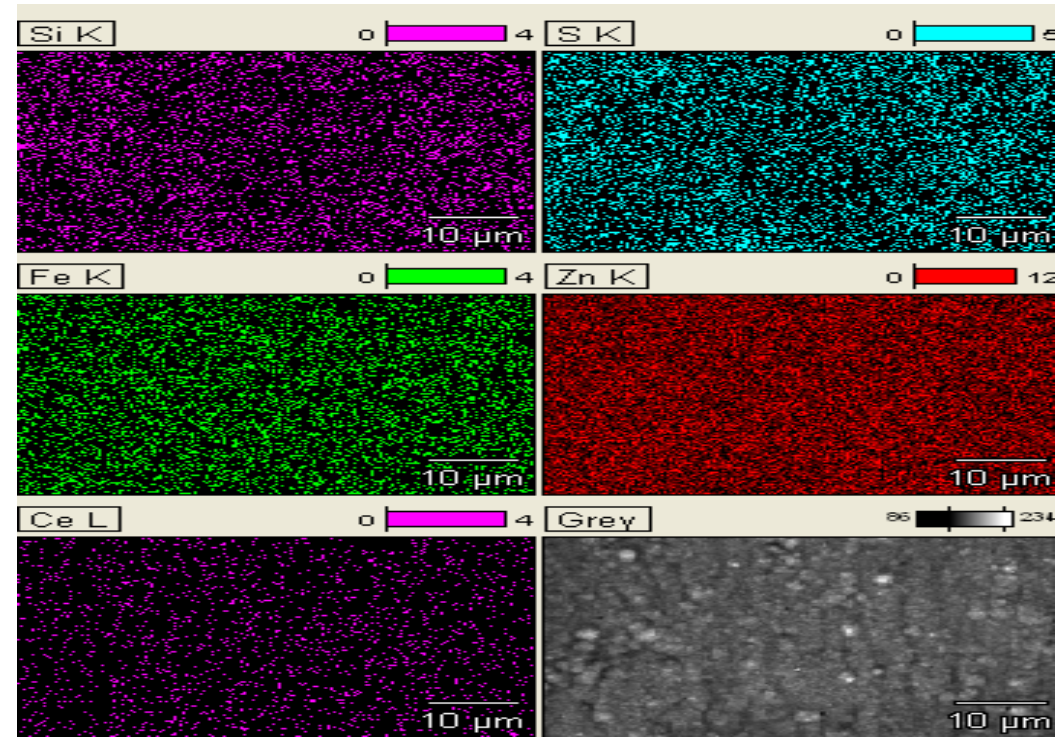

Figura 8: Mapeamento por EDS da superfície da amostra de ZnFe + Ce+ BTSE + BTESPTS antes do ensaio de corrosão. 


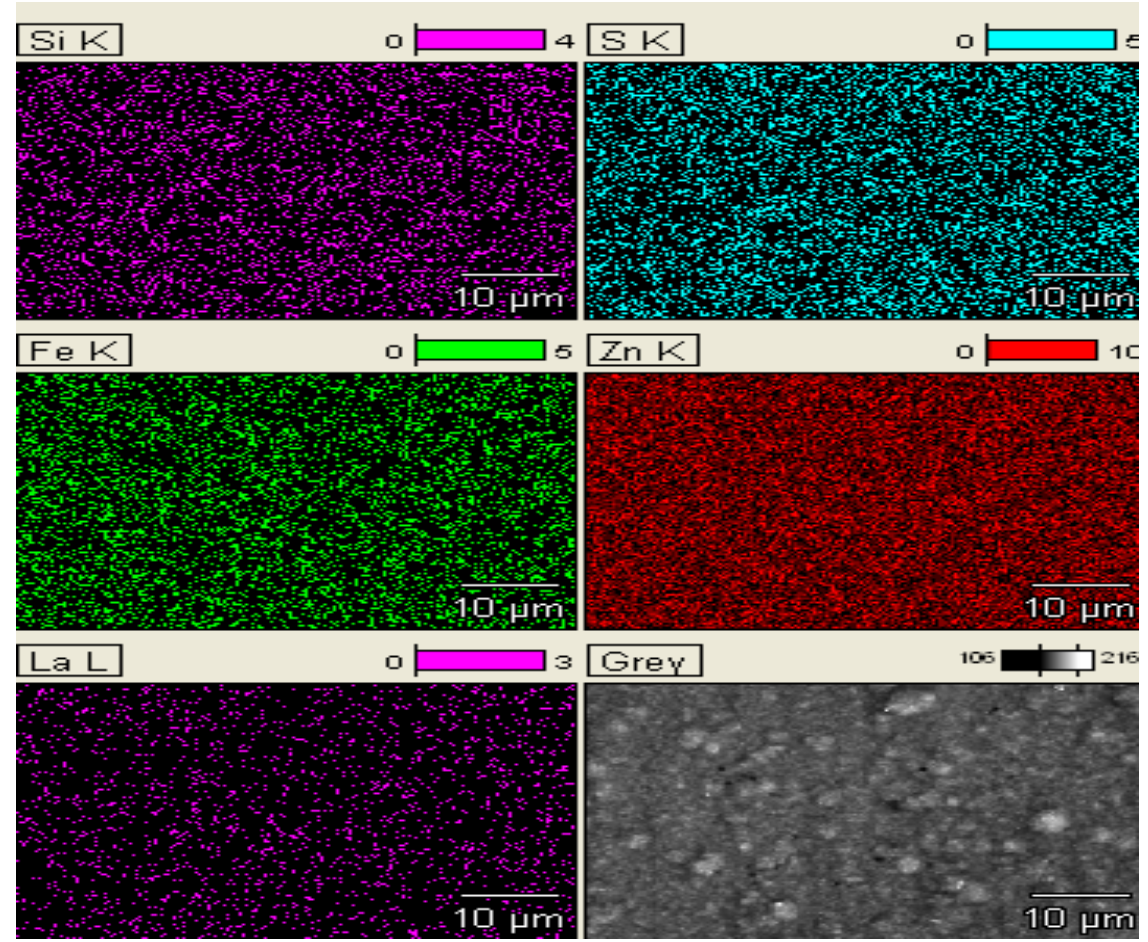

Figura 9: Mapeamento por EDS da superfície da amostra de ZnFe+ La+BTSE+ BTESPTS com novo tratamento antes do ensaio de corrosão.

Os mapeamentos apresentados nas Figuras 8 e 9 são extremamente importantes, pois apresentam distribuição relativa dos elementos detectados por toda a amostra; como pode ser observado essa distribuição é bem homogênea para as amostras.

\section{Imagens do ataque químico dos filmes em solução de $\mathrm{NaCl} 0,05 \mathrm{M}$ por $72 \mathrm{horas}$}

A Figura 10 apresenta as fotografias obtidas com máquina digital comum da liga $\mathrm{ZnFe}$ e das amostras $\mathrm{ZnFe}+$ $\mathrm{Ce}+\mathrm{BTSE}+\mathrm{BTESPT}, \mathrm{ZnFe}+\mathrm{La}+\mathrm{BTSE}+\mathrm{BTESPT}$ antes e depois dos ensaios.

Observa-se que as amostras de $\mathrm{ZnFe+BTSE+BTESPT+Ce} \mathrm{e} \mathrm{ZnFe+BTSE+BTESPT+La} \mathrm{não} \mathrm{apresentam}$ quase nenhum sinal de degradação mesmo após 72horas de ensaio. Para a liga $\mathrm{ZnFe}$ sem os filmes protetores a corrosão de sua superfície é evidente.

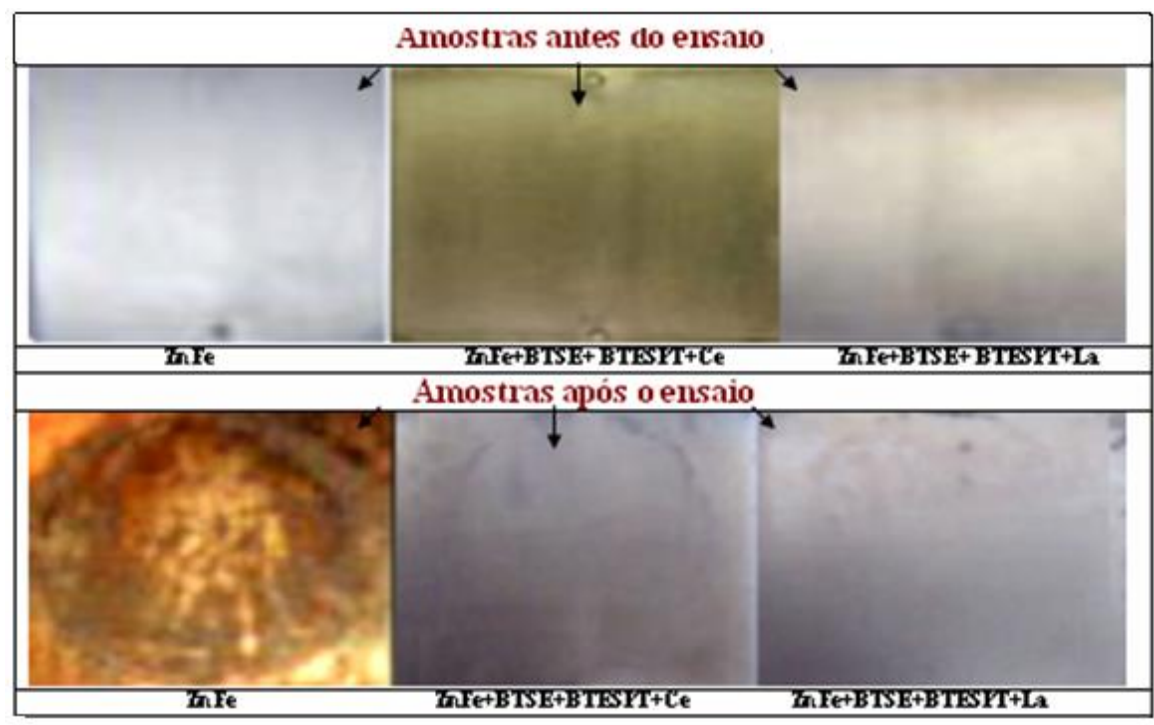

Figura 10 : Fotografias com máquina digital comum da liga $\mathrm{ZnFe}$ e das amostras antes e após 72 horas de ensaio em $\mathrm{NaCl} 0,05 \mathrm{M}$. 


\section{CONCLUSÕES}

O tratamento com BTSE mais BTESPT mais a camada intermediária contendo os óxidos de Ce ou La é melhor que o tratamento com uma só camada de BTESPT mais óxidos de Ce ou La.

Os resultados dos ensaios de polarização mostraram uma diminuição das correntes de resposta nas curvas anódicas e também diminuição das correntes de resposta nas curvas catódicas, sendo que a variação de corrente nos ramos catódico foi mais acentuada, comprovando o efeito barreira dos sistemas contendo camada intermediária de óxidos de terras raras mais silanos estudados.

As curvas de polarização também mostraram que os sistemas contendo óxidos de cério mais silanos são melhores que aqueles contendo óxido de lantânio mais silanos

\section{AGRADECIMENTOS}

Os autores agradecem a CNPq pelo apoio financeiro, e as empresas Tecnorevest e a Momentive pela doação do banho de $\mathrm{ZnFe}$ e das amostras de silanos, respectivamente.

\section{BIBLIOGRAFIA}

[1] SOUZA, M.E.P., Avaliação de Revestimentos Híbridos Organo-inorgânicos e de Resinas Polissiloxanas Aplicadas sobre Aço Galvanizado e sobre Camadas de ZnFe. Tese de D.Sc., Faculdade de Engenharia Mecânica, Universidade Estadual de Campinas, Campinas, SP, Brasil, 2006.

[ 2] CORRÊA A.G., ZUIN V. G., Química Verde: fundamentos e aplicações, vol. 5, São Carlos, Edufscar, 2009.

[ 3] OJEC- Official Journal of the Europe Communities - End-of-Life Vehicles - Directive 2000/53/ec of the European Parliament and of the Concil, 18- setembro 2000.

[4] SANTOS, M.C.G., Estudo de Filmes Poliméricos contendo Silanos $\alpha$-UPS e VS como Revestimentos Promotores de Adesão em Superfícies Galvanizadas e Eletrodepósitos de Ligas $\mathrm{Zn} / \mathrm{Fe}$ e $\mathrm{Zn} / \mathrm{Co}$. Dissertação de M.Sc., Faculdade de Engenharia Mecânica, Universidade Estadual de Campinas, Campinas-SP, 2005.

[5] KASTEN, L. S., GRANT, J. T., GREBASCH., et al., "An XPS study of cerium dopants in sol-gel coatings for aluminum 2024-T3", Surface and Coatings Technology, v. 140, n.1, pp. 11-15, 2001.

[6] GARCIA-HERAS, M., JIMENEZ MORALES A., CASAL, B., et al., "Preparation and electrochemical study of cerium-silica sol-gel thin films", Journal of Alloys and Compounds, v.380, n.1-2, pp. 219-224, 2004.

[7] PENG, S., ZHAO, W., ZENG Z., et al., "Preparation of anticorrosion hybrid silica sol-gel coating using $\mathrm{Ce}(\mathrm{NO} 3) 3$ as catalyst", J. Sol-Gel Sci Technol, v. 66, n.1, pp.133-138, 2013

[8] ARAMAKI, K., "The inhibition effects of cation inhibitors on corrosion of zinc in aerated $0.5 \mathrm{M} \mathrm{NaCl}$ ", Corrosion Science, v. 43, n.8, pp.591-604, 2001.

[9] ARAMAKI, K.., "Preparation of self-healing protective films on a zinc electrode treated in a cerium (III) nitrate solution and modified with sodium phosphate and cerium (III) nitrate". Corrosion Science. v. 46, n.6, pp. 1565-1579, Sep. 2003.

[10] ARAMAKI, K., "Preparation of chromate-free, self-healing polymer films containing sodium silicate on zinc pretreated in a cerium(III) nitrate solution for preventing zinc corrosion at scratches in $0.5 \mathrm{M} \mathrm{NaCl}$ ", Corrosion Science, v. 44, n.6, June 2002, pp. 1375-1389, 2002.

[11] ARAMAKI, K., "Self-healing protective films prepared on zinc electrodes by treatment in a cerium(III) nitrate solution and modification with sodium phosphate and calcium or magnesium nitrate", Corrosion Science, v.45, n.10, pp. 2361-2376, 2003.

[12] ARAMAKI, K., "Prevention of zinc corrosion in oxygenated $0.5 \mathrm{M} \mathrm{NaCl}$ by treatment in a cerium (III) nitrate solution and modification with sodium hexadecanoate", Corrosion Science, v. 48, n.10, pp. 32983308, 2006.

[13] ARAMAKI, K., "Self-healing protective films prepared on zinc by treatments with cerium (III) nitrate and sodium phosphate", Corrosion. Science, v.44, n.11, pp. 2621-2634, 2002. 
[14] ZHU, D., OOIJ, W.J. V., "Corrosion protection of AA 2024-T3 by bis-[3(triethoxysilyl)propyl]tetrasulfide in sodium chloride solution. Part 2: Mechanism for Corrosion Protection", Corrosion Science, v. 45, n.10, pp. 2177-2197, 2003.

[15] FERREIRA, M.G.S., DUARTE, R.G., MONTEMOR, M.F., et al., "Silanes and rare earth salts as chromate replacers for pre-treatments on galvanized steel", Electrochimica acta, v.49, n.17-18, pp.29272935, 2004.

[16] PENG, T., MAN, R., "Rare earth and silane as chromate replacers for corrosion protection on galvanized steel", Journal of rare Earths, v.27, n.1, pp.159-163, 2009.

[17] Aramaki, K., "Self-healing mechanism of an organosiloxane polymer film containing sodium silicate and cerium(III) nitrate for corrosion of scratched zinc surface in $0.5 \mathrm{M} \mathrm{NaCl}$ ", Corrosion Science, v. 44, n.7, pp. 1621-1632, 2002.

[18] TRABELSI, W., CECILIO, P., FERREIRA, M.G.S., et al., "Electrochemical assessment of the selfhealing properties of Ce-doped silane solutions for the pre-treatment of galvanized steel substrates", Progress in Organic Coatings, v.54, n.4, pp. 276-284, 2005.

[19] TRABELSI, W., TRIKI, E., DHOUIBI, L., et al., "The use of pre-treatments based on doped silane solutions for improved corrosion resistance of galvanized steel substrates", Surface \& Coatings Technology, v. 200, pp.4240- 4250, 2006.

[20] SANTOS M.C.G., Avaliação do desempenho de filmes contendo silanos e sais de terras raras para proteção contra corrosão de camadas $\mathrm{ZnFe}$ eletrodepositadas, Tese de D.Sc., Faculdade de Engenharia Mecânica, Universidade Estadual de Campinas, Campinas, SP, Brasil, 2005.

[21] OLIVEIRA, M.F., Estudo da Influência de organo-silanos na resistência à corrosão de aço carbono por meio de técnicas eletroquímicas, Tese de D.Sc., Departamento de Engenharia Metalúrgica e de Materiais, Escola Politécnica da Universidade de São Paulo, São Paulo, SP, Brasil, 2006.

[22] FERREIRA, M.G.S., DUARTE, R.G., MONTEMOR, M.F., et al., "Silanes and rare earth salts as chromate replacers for pré-treatments on galvanized steel", Electrochimica Acta, v.49, n.17-18 pp.2927$2935,2004$.

[23] CABRAL, A., DUARTE, R.G., MONTEMOR, M.F., et al., "Analytical characterisation and corrosion behaviour of bis-[triethoxysilylpropyl]tetrasulphide pre-treated AA2024-T3", Corrosion Science, v.47, n.3, pp. 869-881, 2005

[24] CABRAL, A.M, TRABELSI, W., SERRA, R., et al., "The corrosion resistance of hot dip galvanized steel and AA2024-T3 pre-treated with bis-[triethoxysilylpropyl] tetrasulfide solutions doped with $\mathrm{Ce}\left(\mathrm{NO}_{3}\right)_{3}$ ", Corrosion Science, v.48, n.11, pp. 3470-3758, 2006.

[25] TRABELSI, W.,TRIKI, E., DHOUIBI, L., et al., "The use of pre-treatments based on doped silane solutions for improved corrosion resistance of galvanized steel substrate", Surf. Coat. Technol., pp.42404250, 2006.

[26] ZHU, D., OOIJ, W.J. V., "Enhanced corrosion resistance of AA2024-T3 and hot-dip galvanised steel using mixture of bis-[triethoxysilylpropyl] tetrasulfide and bis-[triethoxysilylpropyl]amine", Electrochimica Acta, v.49, n.7, pp.1113-1125, 2004. 